\title{
Vertebral Osteomyelitis Due to Candida Species: A Cohort Study and Review of the Literature
}

\author{
Amanda Ramos ${ }^{1}$, Paul M. Huddleston ${ }^{2}$, Robin Patel ${ }^{3}$, Emily Vetter ${ }^{3}$, Elie F. Berbari ${ }^{4}$ \\ ${ }^{1}$ Mayo Medical School, Mayo Clinic, Rochester, USA, ${ }^{2}$ Department of Orthopedic Surgery, Mayo Clinic, Rochester, USA, ${ }^{3}$ Department \\ of Laboratory Medicine and Pathology, Mayo Clinic, Rochester, USA, ${ }^{4}$ Division of Infectious Diseases, Mayo Clinic, Rochester, \\ USA. \\ Email: berbari.elie@mayo.edu
}

Received March $7^{\text {th }}, 2013$; revised April 28 ${ }^{\text {th }}, 2013$; accepted May $15^{\text {th }}, 2013$

Copyright (c) 2013 Amanda Ramos et al. This is an open access article distributed under the Creative Commons Attribution License, which permits unrestricted use, distribution, and reproduction in any medium, provided the original work is properly cited.

\begin{abstract}
Objective: To describe the demographics and outcome of patients with candidal vertebral osteomyelitis (CVO). CVO is a rare and frequently misdiagnosed condition. It may lead to destruction of the vertebral bodies, spinal cord compression and neurological deficits. Methods: The medical records of all patients diagnosed with CVO at our institution between 01/01/1990-12/31/2009 were reviewed. The cumulative probability of treatment success was assessed by the Kaplan-Meier survival method. Patients were followed until death, failure, or loss of follow-up. Results: Nine patients developed CVO during the 20 year study period. The cervical spine was involved in 5 cases. Seven patients presented with mechanical-type pain, while 2 patients had an elevated temperature at diagnosis. A contiguous infection between the upper airways and the cervical spine was present in 4 patients. One patient presented with concomitant candidemia. Candida albicans, Candida parapsilosis, and Candida glabrata were cultured in 3 of 9 cases respectively. Eight of 9 were treated with azole-based therapy. Patients were followed for an average of 20 months (range 1 - 75 months). The cumulative incidence of success was $66 \% \pm 19 \%$ at 1 year and $55 \% \pm 20 \%$ at 2 years of follow-up. Conclusions: CVO presents insidiously and is associated with a long duration of symptoms. It most frequently affects the cervical spine and is associated with a poor outcome.
\end{abstract}

Keywords: Vertebral Osteomyelitis; Candida; Back Pain; Antifungal Therapy

\section{Introduction}

Candida vertebral osteomyelitis is a rare and frequently misdiagnosed condition. Since 1970, the number of cases reported in the literature has increased, likely due to the increase in the number of immunocompromised patients [1]. CVO often presents with non-specific physical examination findings. When diagnosed early, CVO is associated with an $85 \%$ cure rate [1]. If diagnosed in later stages, destruction of the vertebral bodies and the resultant bony and epidural abscess cause compression of the spinal cord potentially leading to neurological deficits [1]. The insidious nature of the disease progression, the non-specific clinical signs and laboratory values and the failure to appreciate Candida species as potential pathogens are all likely reasons behind the delay in the diagnosis [2].

Candida species are a group of fungal organisms that are part of the normal human microbiological flora. Infection occurs when host defenses are suppressed and Candida becomes an opportunistic pathogen [3]. There are 150 species of Candida that can cause disease in animals and humans. They are saprophytic organisms that can be cultured from the skin and mucosal membranes of the body. Infections caused by these organisms involve the skin and soft tissues, rarely the bones and joints. The skeletal system may become involved with Candida spp as a result of hematogenous spread from soft tissue abscesses or seeding from a distant infectious focus [3]. This organism commonly localizes to the vertebra or the sternum [3]. Candida spp accounts for the most common fungal etiology of vertebral osteomyelitis. A review of the literature conducted by Miller et al reported that patients with CVO typically undergo prolonged treatment ranging from 1 week to 4 months in duration. Treatment includes the use antifungal therapies such as amphotericin B or azoles. Surgical debridement and spinal stabilization may be required in a subgroup of patients recalcitrant to medical treatment [4].

The presentation of a patient with CVO to our institu- 
tion and the unique case history, clinical and laboratory findings, coupled with their imaging and complex coordination of care stimulated interest for this study. Based on our case experience, we hypothesize that CVO differs substantially in presentation and clinical course from bacterial vertebral osteomyelitis. The authors of this study asked: 1) what is the clinical presentation of CVO and 2) what is the natural history and outcome of the disease? Lastly, how do the patients seen at our institution differ from the patients described in the scientific literature? We therefore reviewed the demographics of all patients with CVO seen at our institution between 01/01/1990-12/31/2009 with special interest on the outcomes.

\section{Materials and Methods}

\subsection{Patients}

The study was approved by our institutional review board (IRB\# 10-002361). We retrospectively evaluated the clinical and diagnostic findings, treatment and outcomes of 9 patients with CVO. We reviewed all cases of spine infection (vertebral osteomyelitis, discitis, epidural abscesses) in patients seen at the Mayo Clinic Rochester between 1/1/1990 and 12/31/2009 by searching the Mayo Clinic microbiological databases for positive spine cultures for Candida spp. Patients 18 years or older with positive candidal cultures from the vertebral body, intervertebral disc or paraspinal fluid were included in the study. Cases of CVO and discitis were defined as a consistent clinical picture, compatible radiological findings, and positive bone, disc or paraspinal fluid cultures for candida. This search identified 9 patients, 6 of whom were male and 3 female. Both monomicrobial and polymicrobial infections containing Candida spp were included in this study.

Medical and surgical diagnostic approaches and therapies were not standardized and were performed at the discretion of the treating physicians. We collected clinical data (laboratory values, radiographic findings, histopathology results) and the clinical outcome at the time of last follow up. Data collection was standardized by using a coded data collection instrument. A Kaplan Meier survival curve was used to depict outcomes amongst the cohort patients. A positive outcome was defined as a resolution of symptomology with a negative work-up for relapse of vertebral osteomyelitis due to candida. A negative outcome was defined as microbiological relapse, radiological relapse, return of symptomology or loss of follow-up or death.

\subsection{Literature Review}

We conducted a MEDLINE search of the English literature from 1970 to 2010 and a bibliographic search of published articles to identify cases of candidal vertebral osteomyelitis. Keywords used included candida vertebral osteomyelitis, discitis, epidural abscess and spondylodiscitis. We reviewed all data available including clinical presentation, diagnostic techniques, therapeutic approaches and patient outcomes. To identify the cases as $\mathrm{CVO}$ or discitis we required clinical suspicion of vertebral osteomyelitis/discitis with a positive candidal culture from a spinal biopsy. Similar to the cohort patients, the data collection was standardized using a coded data collection instrument.

\section{Results}

\subsection{Patient Cohort}

In our patient cohort, the average age of diagnosis was 57 years of age (Table 1). Two patients had a previous history of osteomyelitis occurring in the cervical spine and in association with contamination of spinal instrumentation. On average, our CVO patients had 5 documented co-morbid conditions. Prior endovascular infections were observed in 3 patients. One of the 3 individuals with a line-related infection grew Candida spp and a second patient grew yeast of unspecified species, resulting in two patients with a known prior candidemia. One of these patients developed CVO as a result of the candidemia. Five out of the 9 patients were on antimicrobial therapy within 2 weeks of their CVO diagnosis. Lastly, 6 patients presented with a polymicrobial infection and 3 presented with a mono-candidal infection.

Four of the 9 patients presented with mechanical-type back pain and 2 patients with mechanical neck pain. Two patients presented with radicular type pain, one with both radicular and mechanical pain and 5 had sinus tract formation around the infected vertebral bodies. Neurological symptoms secondary to CVO were found in 3 patients; one experienced weakness of the upper and lower extremities bilaterally, one patient experienced decreased sensation on the left side of the body and one patient had documented hyperreflexia and positive Babinski signs bilaterally with no motor weakness noted on exam. The average duration of symptoms to the time of diagnosis was 4.8 months. Two patients were febrile at presentation. Five patients had evidence of a fistulous connection between the gastrointestinal tract and the spinal column. Six patients presented with multi-level vertebral body and/or disc involvement. Six patients displayed radiologic findings of extra-spinal manifestations of infection. These findings included paraspinal abscesses, epidural abscesses, and psoas muscle involvement. The average erythrocyte sedimentation rate at diagnosis was $62.25 \mathrm{~mm} / \mathrm{h}$ (range $24 \mathrm{~mm} / \mathrm{h}$ to $92 \mathrm{~mm} / \mathrm{h}$ ). The average white blood cell count was $8.3625 \times 10^{9} / \mathrm{L}$ (range $5.8 \times$ $10^{9} / \mathrm{L}$ to $21.2 \times 10^{9} / \mathrm{L}$ ) and the average C-reactive protein 


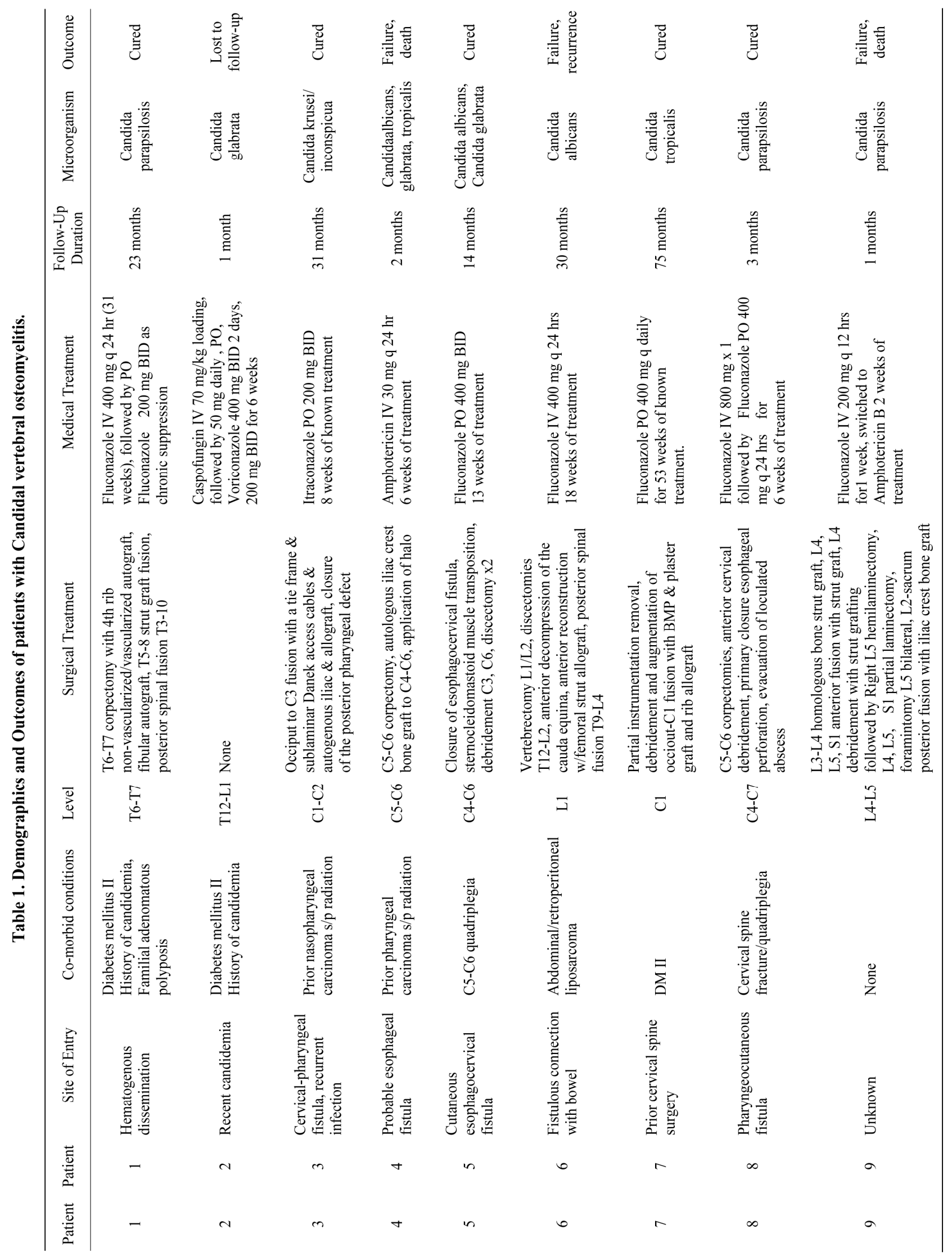


was $11.2 \mathrm{mg} / \mathrm{L}$ (range 14.7 to 28.3) [2].

Eight patients underwent surgery. The indications included correction of neurological deficits, spinal instability, debridement of spinal instrumentation, persistent pain, drainage of abscesses and correction of gastrointestinal fistulas. Bone grafts were placed in 4 patients. On average, patients received medical therapy for 16.6 weeks (range 16 days to 53 weeks). Among those patients with a good outcome the average duration of treatment was 24 weeks with 2 patients on chronic oral suppression.

Therapy was effective in 5 patients with documented follow up. During the course of follow-up, 1 patient had enhancement of an epidural abscess on MRI and 6 out of the 9 patients had persistent pain requiring the use of analgesics. After 1 year and 2 years of follow up, 66\% and 55\% had a successful outcome respectively (Figure 1). Of the patients with negative outcomes, 1 patient was lost to follow-up and 2 patients died. Following a course of antifungal therapy and surgery, 1 patient relapsed with positive paraspinal cultures.

\subsection{Literature Review}

Our review of the English literature revealed 104 cases of CVO. The average age at diagnosis of CVO was 53.9 years of age. Thirty five percent of patients had priorcandidemia and $30 \%$ of patients had an indwelling central venous catheter. Fifty one percent of patients had documented prior antimicrobial use and $10.5 \%$ of patients were on systemic steroids. Sixty-eight percent of patients were immunocompromised.

Seventeen percent of patients presented with fever and another $17 \%$ presented with neurological symptoms. Forty-one percent of the documented cases affected the lumbar spine, $28 \%$ the thoracic spine, $6.3 \%$ the cervical spine, and $12 \%$ had multiple involvement. Fifteen percent of patients had epidural abscesses, $42 \%$ presented with an elevated erythrocyte sedimentation rate, $14 \%$ patients had a known elevated C-reactive protein and $6.7 \%$ had an elevated white blood cell count. Thirty-six patients had a documented candidemia [5-29]. Fortythree percent of CVO cases were due to Candida albicans. C. tropicalis and C glabrata caused CVO in $12.5 \%$ and $5.7 \%$ of cases respectively. C. parapsilosis. C. krusei, C. paratropicalis, C. hyphae, and C. pseudotropicalis were rarely associated with CVO. Seventy-five percent of patients had a successful outcome [30]. The average duration of medical therapy in the literature was 17.6 weeks. In those patients who were cured of the disease, the average duration of known treatment was 19.8 weeks.

The average duration of follow up for the documented cases was 13.3 months.

\section{Discussion}

The purpose of this study was to analyze the clinical features and outcomes of patients with candida vertebral osteomyelitis (CVO) seen at our institution and to review the literature on this topic.

Similarities in clinical presentation and natural history of the disease were observed between the two groups.

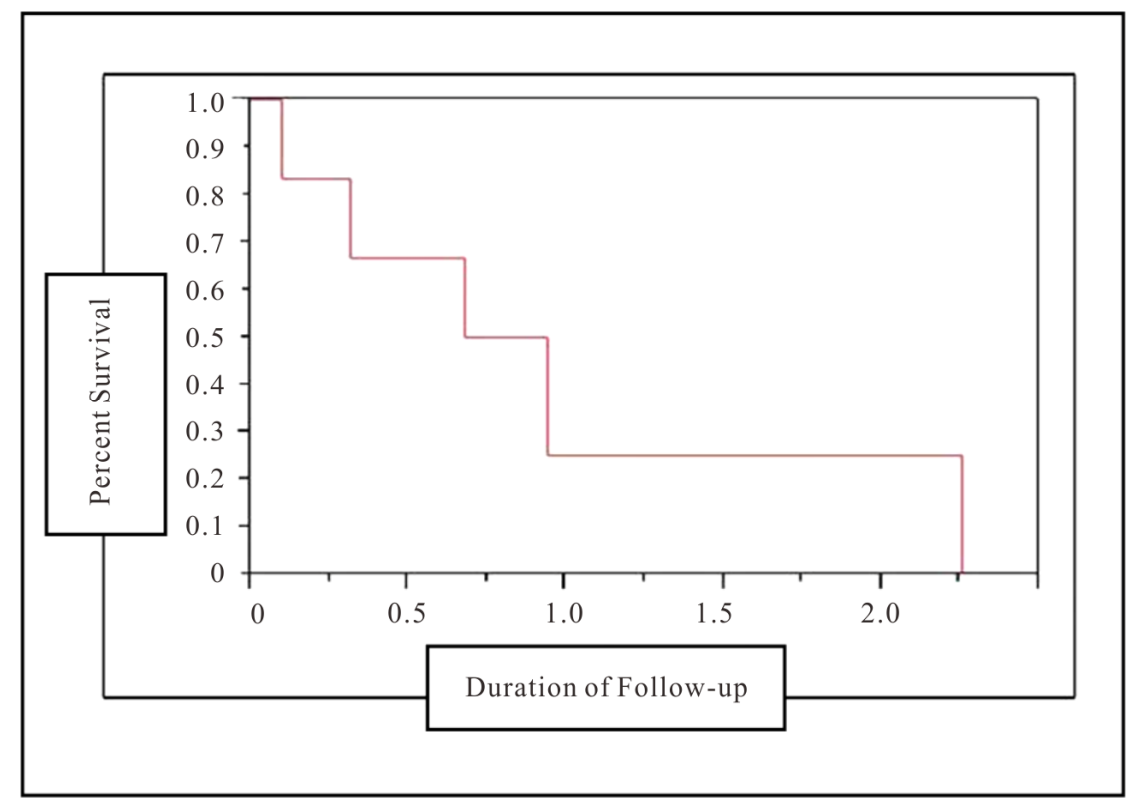

Figure 1. Outcomes of candida vertebral osteomyelitis patients. A Kaplan Meier survival curve depicting percent survival (y-axis) and duration of follow-up (x-axis) in the cohort patients. At 1 year of follow-up, 66\% of patients had survived, at 2 years of follow-up, $55 \%$ of the cohort patients had survived 
Physical exam findings in our cohort tended to be nonspecific, consisting of chronic insidious mechanical back pain, coupled with radicular manifestations. Our findings are consistent with a review of the published literature performed in 2001 describing 59 cases of CVO. In this study, $95 \%$ of the reported cases had thoracic and lumbosacral disease involvement. Eighty three percent had symptoms of back pain, 32\% had fever and 19\% had neurological defects [4]. Other similarities observed between our patient cohort and the literature patients included an elevated erythrocyte sedimentation rate and c-reactive protein at presentation and normal white blood cell count $[3,31]$. The majority of patients were afebrile at presentation. Lastly, the main organism isolated from diagnostic spinal biopsies was Candida albicans. These results are consistent with a review of the literature by Miller et al that identified Candida albicans as the causative agent in the majority of CVO cases (62\% of patients). Similarities between the literature and our cohort demonstrated that CVO often occurs in immunocompromised patients [4-11,31-36]. The main co-morbid conditions identified in both the literature review and our cohort were diabetes mellitus, malignancies and prior surgeries involving upper airways or the esophagus. These findings suggest that patients presenting with indolent, persistent, back pain and who have received radiation or chemotherapy for upper airway or esophageal malignancies, should be evaluated for the possibility of CVO [12-28,37-60].

Several differences were also identified between our cohort and the literature review. CVO is typically the result of hematogenous seeding of the vertebral bodies from a prior candidemia caused by contamination of an indwelling catheter or inoculation into the blood by injectable drugs [3]. In contrast, our cohort demonstrated several examples of direct contiguous spread from gastrointestinal or upper airway fistulous connections with the spine. As indicated in the results section of this article, several of our patients had persistent esophageal or other upper gastrointestinal-cutaneous fistulas. These fistulas were caused by prior nasopharyngeal carcinoma, previous surgeries to the area and prior radiation therapy. Although the literature describes the lumbar spine as the most common level affected, the majority of our patients had cervical spine involvement; a finding due to fistulous tract formation between the pharyngeal structures and the cervical spine, thought to be secondary to radiation and oncologic surgical treatment [1]. Lastly, in our cohort most patients with CVO were not candidemic suggesting a contiguous source of infection.

The most common diagnostic techniques used in the detection of CVO were MRI followed by CT-guided biopsy and surgical biopsy. Radiological findings tend to be non-specific and are very similar to vertebral osteo- myelitis caused by other microorganisms [3]. According to Williams et al, typical MRI findings for CVO can be seen in both T1 and T2 weighted images as an absence of disc hypersensitivity with preservation of the internuclear cleft on T2-weighted images [33]. Other non-specific findings may demonstrate progressive destruction of the vertebral bodies, irregular definition of the end plates with narrowing of the disc space and several lesions in

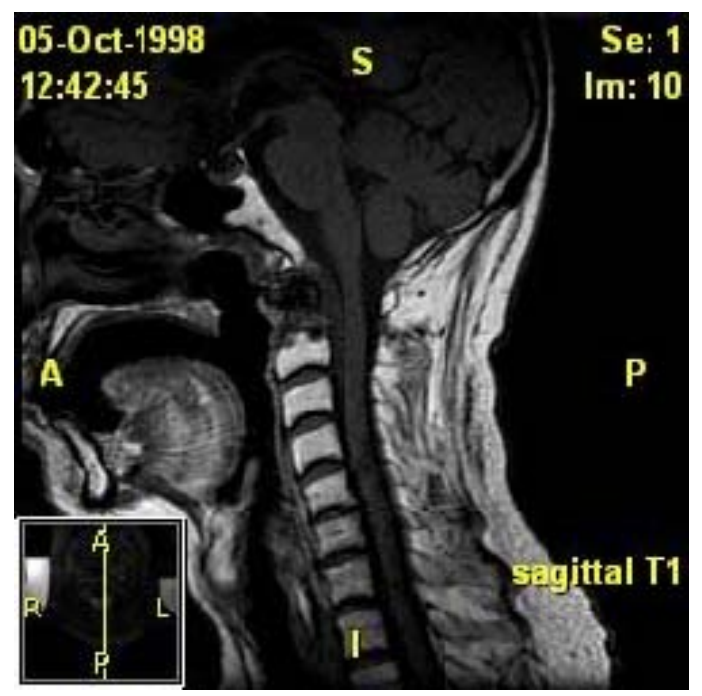

(a)

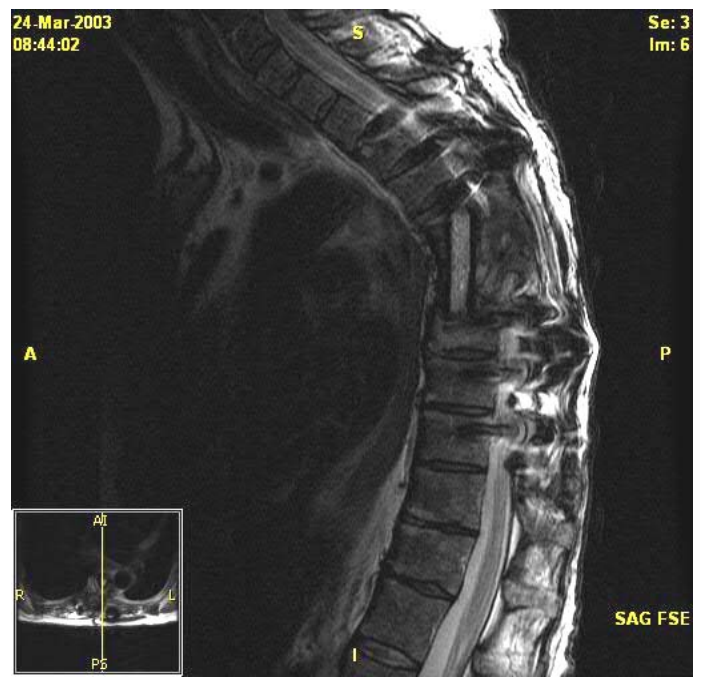

(b)

Figure 2. (a) MRI of the thoracic spine with contrast. Patient 1 pre-operative image. There is near-complete destruction of the T6 vertebral body with loss of the T7 vertebral height. There is abnormal signal and enhancement within the residual T6 and T7 vertebral bodies including the endplates and intervening disk. There is bilateral paraspinous enhancing tissue from the upper T5 through the T8 level; (b) MRI thoracic spine with contrast. Patient 1 post-operative image, s/p initiation of treatment. Posterior spinal fusion with hardware T3 to T10 with T6-T7 corpectomy and T5T8 strut graft fusion. 


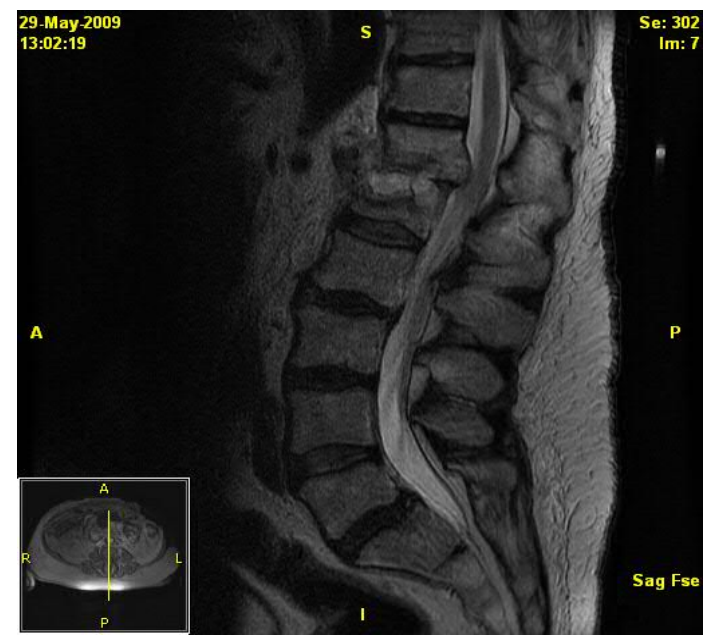

Figure 3. MRI of the lumbar spine without contrast. Patient 2 pre-treatment. Destructive changes at the T12-L1 interspace and $\mathrm{T} 12$ and $\mathrm{L} 1$ vertebral bodies. There are surrounding inflammatory changes in the paraspinal musculature. Soft tissue extends in the left epidural space from the upper T12 vertebral body to the T12-L1 interspace with extension into, and effacement of, the left T12-L1 neural foramen.

the bones with no bone marrow involvement (Figures 2-4) $[2,4,41]$. As noted in the results section, extension of the CVO infection to the epidural, paraspinal space, or to the psoas muscle was observed in several patients; the most common extra-vertebral manifestation of infection was epidural abscess. This result was also noted in the literature patients [2,5,68,17,24,27,32,34,37,39,41,61,62]. Therefore, it is important to recognize the possibility of extraspinal manifestations of infection when considering treatment options.

As specified in Table 1, patients with CVO are treated with a combination of antifungal therapy and surgical debridement and/or stabilization. This strategy is associated with the highest cure rate in the literature and in our cohort. Azole-based therapy or Amphotericin-based antifungal therapy is the treatment of choice in both groups. All of our patients with successful outcomes were treated with azole therapy as the only anti-fungal medication. Current treatment recommendations published by the Infectious Diseases Society of America (IDSA) suggest fluconazole $400 \mathrm{mg}$ (6 mg/kg) daily for 6 - 12 months or lipid formulation amphotericin B, 3 - $5 \mathrm{mg} / \mathrm{kg}$ daily for several weeks, then fluconazole for 6 - 12 months. An alternative therapeutic approach is to use echinocandin or amphotericin B dexoycholate 0.5 - $1 \mathrm{mg} / \mathrm{kg}$ daily for several weeks followed by fluconazole for 6 - 12 months. The IDSA also suggests that surgical intervention is often necessary. Based on the outcomes of our patient cohort and the literature patients, we recommend treating CVO for a minimum of 20 - 23 weeks as this was the average length of time that the patients with successful

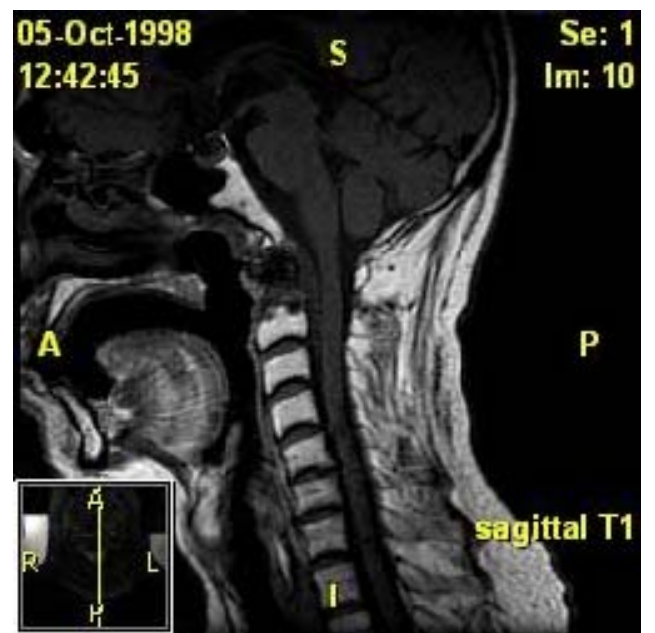

(a)

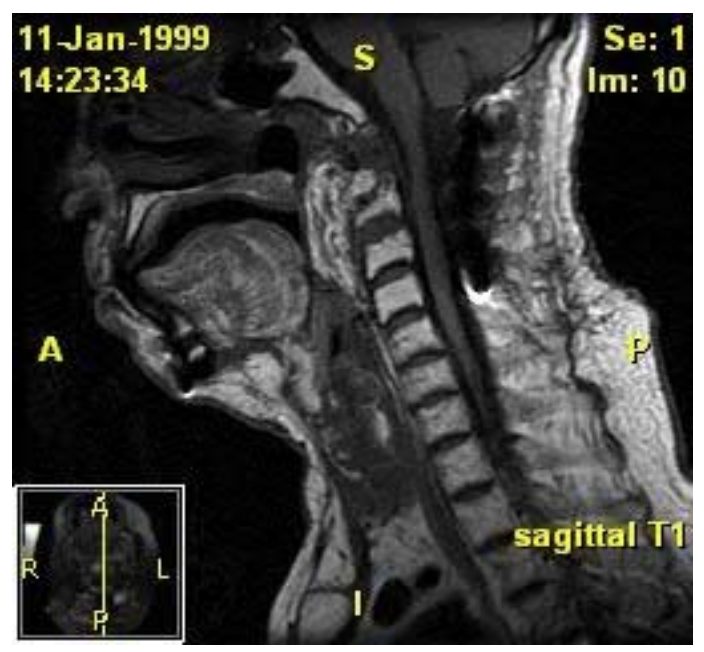

(b)

Figure 4. (a) MRI of the soft tissues of the neck and cervical spine. Patient 3 pre-operative image. The examinations demonstrate lytic destruction of the anterior arch of $\mathrm{C1}$, base of the dens and superior right aspect of the body of $\mathrm{C}-2$. There is a large ulcerated defect within the nasopharynx at and to the right of midline which is contiguous with this defect with air or gas extending into the bony defect. There are also multiple small bubbles of gas within the ventral epidural space from $\mathrm{C}-1$ through the lower body of $\mathrm{C}-2$. There is an associated peripherally enhancing ventral epidural collection extending from the foramen magnum to the mid body of C-3 which results in effacement of the ventral subarachnoid space but no definite cord deformity; (b) MRI of the skull base, face, and neck. Patient 3 post-operative image. A free flap reconstruction of the posterior nasopharyngeal wall and is seen on this MRI. Edema involving the soft palate, oro- and hypopharynx, and larynx is also seen.

outcomes underwent treatment. Surgical intervention is also recommended when feasible. While fluconazole therapy side-effects include various interactions with other medications, nausea, vomiting, and rare complica- 
tions such as agranulocytosis, fulminant liver failure and Steven Johnson Syndrome, all of our cohort patients tolerated the medication well. Servalli et al. recommends continuing therapy until the erythrocyte sedimentation rate normalizes [63].

Compared to the findings of this present study, the literature describes a high percentage of patients with successful outcomes. Our study identified a 55\% cumulative incidence of successful outcome after two years of follow up. The discrepancy between the success rate described in the literature and that described in our present study may be explained by the limited duration of follow-up examination in the literature and the propensity for publication bias of successfully treated case reports as well as the difference in the definition of a successful outcome. In these cases there was a 13 month length of follow up duration on average. In our cohort the average duration of follow up was 20 months. The poor outcome seen in our patient cohort may also be explained by the small nature of the study and a biased representation of the larger population of patients with CVO.

The major limitations to our study are inherent to its retrospective nature and small sample size. This is a function of the low incidence of the disease. A review of the literature was conducted for the purpose of analyzing the patient population in its entirety to supplement our small cohort of patients. Since our data was collected from a single tertiary referral medical center there is a potential for a referral bias.

In conclusion, CVO should be suspected in patients with immunosuppression or chronic illness presenting with persistent back pain, decreased range of motion and neurological deficits [4-11,31-36]. Fever is not commonly observed, however an elevated ESR and CRP is common. MRI followed by CT-guided biopsy or an open biopsy are often used to confirm the diagnosis of CVO. Treatment often requires a combination of medical and surgical approaches. Both azoles and Amphotericin B can be used for medical therapy.

\section{REFERENCES}

[1] R. M. Khazim, U. K. Debnath and Y. Fares, "Candida albicans Osteomyelitis of the Spine: Progressive Clinical and Radiological Features and Surgical Management in Three Cases," European Spine Journal, Vol. 15, No. 9, 2006, pp. 1404-1410. doi:10.1007/s00586-005-0038-z

[2] N. Ozdemir, L. Celik, S. Oguzoglu, L. Yildirim and H. Bezircioglu, "Cervical Vertebral Osteomyelitis and Epidural Abscess Caused by Candida albicans in a Patient with Chronic Renal Failure,” Turkish Neurosurgery, Vol. 18, No. 2, 2008, pp. 207-210.

[3] J. G. Cha, H. S. Hong, Y. W. Koh, H. K. Kim and J. M. Park, "Candida albicans Osteomyelitis of the Cervical Spine,” Skeletal Radiology, Vol. 37, No. 4, 2008, pp. 347350. doi:10.1007/s00256-007-0429-9
[4] D. J. Miller and G. C. Mejicano, "Vertebral Osteomyelitis Due to Candida Species: Case Report and Literature Review,” Clinical Infectious Diseases, Vol. 33, No. 4, 2001, pp. 523-530. doi:10.1086/322634

[5] J. Garbino, I. Schnyder, D. Lew, K. Bouchuiguir-Wafa and P. Rohner, "An Unusual Cause of Vertebral Osteomyelitis: Candida Species,” Scandinavian Journal of Infectious Diseases, Vol. 35, No. 4, 2003, pp. 288-291. doi:10.1080/00365540310000067

[6] M. P. Curran and L. G. Lenke, "Torulopsis Glabrata Spinal Osteomyelitis Involving Two Contiguous Vertebrae. A Case Report,” Spine, Vol. 21, No. 7, 1996, pp. 866-870. doi:10.1097/00007632-199604010-00019

[7] C. Ferra, B. N. Doebbeling, R. J. Hollis, M. A. Pfaller, C. K. Lee and R. D. Gingrich, "Candida Tropicalis Vertebral Osteomyelitis: A Late Sequela of Fungemia,” Clinical Infectious Diseases, Vol. 19, No. 4, 1994, pp. 697-703. doi:10.1093/clinids/19.4.697

[8] P. G. Owen, B. K. Willis and E. C. Benzel, "Torulopsis Glabrata Vertebral Osteomyelitis,” Journal of Spinal Disorders, Vol. 5, No. 3, 1992, pp. 370-373. doi:10.1097/00002517-199209000-00018

[9] L. C. Almekinders and W. B. Greene, "Vertebral Candida Infections. A Case Report and Review of the Literature," Clinical Orthopaedics and Related Research, Vol. 267, 1991, pp. 174-178.

[10] I. Potasman, Z. Leibovitz and M. Sharf, "Candida Sepsis in Pregnancy and the Postpartum Period," Reviews of Infectious Diseases, Vol. 13, No. 1, 1991, pp. 146-149. doi:10.1093/clinids/13.1.146

[11] S. C. Imahori, T. Papademetriou and D. M. Ogliela, “Torulopsis Glabrata Osteomyelitis. A Case Report,” Clinical Orthopaedics and Related Research, Vol. 219, 1987, pp. 214-220.

[12] J. Bruns, T. Hemker and G. Dahmen, "Fungal Spondylitis. A Case of Torulopsis glabrata and Candida tropicalis Infection,” Acta Orthopaedica Scandinavica, Vol. 57, No. 6, 1986, pp. 563-565. doi:10.3109/17453678609014795

[13] B. S. Shaikh, P. C. Appelbaum and R. C. Aber, "Vertebral Disc Space Infection and Osteomyelitis Due to Candida albicans in a Patient with Acute Myelomonocytic Leukemia," Cancer, Vol. 45, No. 5, 1980, pp. 1025-1028. doi:10.1002/1097-0142(19800301)45:5<1025::AID-CNC R2820450532>3.0.CO;2-I

[14] D. P. Eisen, R. MacGinley, B. Christensson, L. Larsson and M. L. Woods, "Candida Tropicalis Vertebral Osteomyelitis Complicating Epidural Catheterisation with Disease Paralleled by Elevated D-Arabinitol/L-Arabinitol Ratios,” European Journal of Clinical Microbiology \& Infectious Diseases, Vol. 19, No. 1, 2000, pp. 61-63. doi:10.1007/s100960050013

[15] F. A. Corso, D. B. Shaul and B. M. Wolfe, "Spinal Osteomyelitis after TPN Catheter-Induced Septicemia," Journal of Parenteral and Enteral Nutrition, Vol. 19, No. 4, 1995, pp. 291-295. doi:10.1177/0148607195019004291

[16] R. F. Mullins, J. M. Still Jr., J. Savage, J. B. Davis and E. J. Law, "Osteomyelitis of the Spine in a Burn Patient Due to Candida albicans,” Burns, Vol. 19, No. 2, 1993, pp. 
174-176. doi:10.1016/0305-4179(93)90045-A

[17] A. M. Sugar, C. Saunders and R. D. Diamond, "Successful Treatment of Candida Osteomyelitis with Fluconazole. A Noncomparative Study of Two Patients," Diagnostic Microbiology and Infectious Disease, Vol. 13, No. 6, 1990, pp. 517-520. doi:10.1016/0732-8893(90)90084-9

[18] J. E. Edwards, S. B. Turkel, H. A. Elder, R. W. Rand and L. B. Guze, "Hematogenous Candida Osteomyelitis. Report of Three Cases and Review of the Literature,” American Journal of Medicine, Vol. 59, No. 1, 1975, pp. 89-94. doi:10.1016/0002-9343(75)90325-3

[19] B. A. Dijkmans, M. I. Koolen, R. P. Mouton, T. H. Falke, P. J. van den Broek and J. W. van der Meer, "Hematogenous Candida Vertebral Osteomyelitis Treated with Ketoconazole,” Infection, Vol. 10, No. 5, 1982, pp. 290-292. doi:10.1007/BF01640877

[20] W. S. Hayes, R. A. Berg, H. D. Dorfman and M. T. Freedman, "Case Report 291. Diagnosis: Candida Discitis and Vertebral Osteomyelitis at L1-L2 from Hematogenous Spread," Skeletal Radiology, Vol. 12, No. 4, 1984, pp. 284-287. doi:10.1007/BF00349511

[21] C. Hennequin, P. Bouree, C. Hiesse, B. Dupont and B. Charpentier, "Spondylodiskitis Due to Candida albicans: Report of Two Patients Who Were Successfully Treated with Fluconazole and Review of the Literature," Clinical Infectious Diseases, Vol. 23, No. 1, 1996, pp. 176-178. doi:10.1093/clinids/23.1.176

[22] S. M. Rachapalli, R. Malaiya, T. A. Mohd and R. A. Hughes, "Successful Treatment of Candida Discitis with 5-Flucytosine and Fluconazole,” Rheumatology International, Vol. 30, No. 11, 2010, pp. 1543-1544. doi:10.1007/s00296-009-1215-X

[23] J. Peman, I. Jarque, M. Bosch, E. Canton, M. Salavert, R. de Llanos, et al., "Spondylodiscitis Caused by Candida Krusei: Case Report and Susceptibility Patterns,” Journal of Clinical Microbiology, Vol. 44, No. 5, 2006, pp. 19121914. doi:10.1128/JCM.44.5.1912-1914.2006

[24] S. L. Chia, B. H. Tan, C. T. Tan and S. B. Tan, “Candida Spondylodiscitis and Epidural Abscess: Management with Shorter Courses of Anti-Fungal Therapy in Combination with Surgical Debridement,” Journal of Infection, Vol. 51, No. 1, 2005, pp. 17-23. doi:10.1016/j.jinf.2004.08.020

[25] T. Tokuyama, S. Nishizawa, N. Yokota, S. Ohta, T. Yokoyama and H. Namba, "Surgical Strategy for Spondylodiscitis Due to Candida albicans in an Immunocompromised Host,” Neurologia Medico-Chirurgica (Tokyo), Vol. 42, No. 7, 2002, pp. 314-317. doi:10.2176/nmc.42.314

[26] G. D. Sebastiani and F. Galas, "Spondylodiscitis Due to Candida Tropicalis as a Cause of Inflammatory Back Pain," Clinical Rheumatology, Vol. 20, No. 6, 2001, pp. 435-437. doi:10.1007/s100670170011

[27] N. J. Dailey and E. J. Young, “Candida glabrata Spinal Osteomyelitis," The American Journal of the Medical Sciences, Vol. 341, No. 1, 2011, pp. 78-82. doi:10.1097/MAJ.0b013e3181f6c6ea

[28] K. Dwyer, M. McDonald and T. Fitzpatrick, "Presentation of Candida glabrata Spinal Osteomyelitis 25 Months after Documented Candidaemia,” Australian \& New Zealand Journal of Medicine, Vol. 29, No. 4, 1999, pp. 571572. doi:10.1111/j.1445-5994.1999.tb00767.x

[29] W. Y. Yu, C. Siu, P. C. Wing, J. F. Schweigel and N. Jetha, "Percutaneous Suction Aspiration for Osteomyelitis. Report of Two Cases,” Spine, Vol. 16, No. 2, 1991, pp. 198-202.

[30] P. G. Pappas, C. A. Kauffman, D. Andes, D. K. Benjamin Jr., T. F. Calandra, J. E. Edwards Jr., et al., "Clinical Practice Guidelines for the Management of Candidiasis: 2009 Update by the Infectious Diseases Society of America,” Clinical Infectious Diseases, Vol. 48, No. 5, 2009, pp. 503-535. doi:10.1086/596757

[31] R. H. Bhogal, M. Nayeemuddin, I. Akhtar, M. Grainger and R. Downing, "Continued Lumbar Spinal Erosion after Repair of Chronic Contained Rupture of a Mycotic Abdominal Aortic Aneurysm,” Surgical Infections, Vol. 9, No. 4, 2008, pp. 475-480. doi:10.1089/sur.2007.054

[32] S. Metcalfe and C. Morgan-Hough, "Cervical Epidural Abscess and Vertebral Osteomyelitis Following NonTraumatic Oesophageal Rupture: A Case Report and Discussion,” European Spine Journal, Vol. 18, No. Suppl 2, 2009, pp. 224-227. doi:10.1007/s00586-009-0889-9

[33] R. L. Williams, M. B. Fukui, C. C. Meltzer, A. Swarnkar, D. W. Johnson and W. Welch, "Fungal Spinal Osteomyelitis in the Immunocompromised Patient: MR Findings in Three Cases," American Journal of Neuroradiology, Vol. 20, No. 3, 1999, pp. 381-385.

[34] P. L. Munk, M. J. Lee, P. Y. Poon, J. X. O’Connell, D. B. Coupland, D. L. Janzen, et al., "Candida Osteomyelitis and Disc Space Infection of the Lumbar Spine,” Skeletal Radiology, Vol. 26, No. 1, 1997, pp. 42-46. doi:10.1007/s002560050189

[35] T. J. Neale, J. C. Muir, H. Mills, J. G. Horne and M. R. Jones, “Candida albicans Vertebral Osteomyelitis in Chronic Renal Failure,” Postgraduate Medical Journal, Vol. 63, No. 742, 1987, pp. 695-698. doi:10.1136/pgmj.63.742.695

[36] G. Ackerman and J. C. Bayley, “Candida Albicans Osteomyelitis in a Vertebral Body previously Infected with: Serratia Marcescens,” Spine, Vol. 15, No. 12, 1990, pp. 1362-1363. doi:10.1097/00007632-199012000-00024

[37] Z. Shaikh, S. Shaikh, F. Pujol, D. Trauber and M. Sam, "Candida Tropicalis Osteomyelitis: Case Report and Review of Literature," American Journal of Medicine, Vol. 118, No. 7, 2005, pp. 795-798. doi:10.1016/j.amjmed.2004.11.027

[38] J. V. Hirschmann and E. D. Everett, “Candida Vertebral Osteomyelitis,” The Journal of Bone \& Joint Surgery, Vol. 58, No. 4, 1976, pp. 573-575.

[39] L. A. Cone, L. Dreisbach, P. Dreisbach and M. Wuesthoff, "Another Patient with Candida Vertebral Osteomyelitis Treated with Liposomal Amphotericin B," Surgical Neurology, Vol. 63, No. 6, 2005, p. 592. doi:10.1016/j.surneu.2005.02.001

[40] A. Schilling, M. Seibold, V. Mansmann and B. Gleissner, "Successfully Treated Candida Krusei Infection of the Lumbar Spine with Combined Caspofungin/Posaconazole 
Therapy,” Medical Mycology, Vol. 46, No. 1, 2008, pp. 79-83. doi:10.1080/13693780701552996

[41] L. A. Cone, R. G. Byrd, B. E. Potts and M. Wuesthoff, "Diagnosis and Treatment of Candida Vertebral Osteomyelitis: Clinical Experience with a Short Course Therapy of Amphotericin B Lipid Complex," Surgical Neurology, Vol. 62, No. 3, 2004, pp. 234-237. doi:10.1016/j.surneu.2003.11.018

[42] M. M. El-Zaatari, K. Hulten, Y. Fares, A. Baassiri, M. Balkis, A. Almashhrawi, et al., "Successful Treatment of Candida Albicans Osteomyelitis of the Spine with Fluconazole and Surgical Debridement: Case Report," Journal of Chemotherapy, Vol. 14, No. 6, 2002, pp. 627-630.

[43] A. Lafont, A. Olive, M. Gelman, J. Roca-Burniols, R. Cots and J. Carbonell, "Candida Albicans Spondylodiscitis and Vertebral Osteomyelitis in Patients with Intravenous Heroin Drug Addiction. Report of 3 New Cases," The Journal of Rheumatology, Vol. 21, No. 5, 1994, pp. 953-956.

[44] B. C. Friedman and G. L. Simon, "Candida Vertebral Osteomyelitis: Report of Three Cases and a Review of the Literature,” Diagnostic Microbiology and Infectious Disease, Vol. 8, No. 1, 1987, pp. 31-36. doi:10.1016/0732-8893(87)90044-7

[45] M. J. Diament, M. Weller and R. Bernstein, "Candida Infection in a Premature Infant Presenting as Discitis," Pediatric Radiology, Vol. 12, No. 2, 1982, pp. 96-98. doi:10.1007/BF00972443

[46] A. J. Thurston and W. J. Gillespie, “Torulopsis Glabrata Osteomyelitis of the Spine: A Case Report and Review of the Literature," Australian and New Zealand Journal of Surgery, Vol. 51, No. 4, 1981, pp. 374-376. doi:10.1111/j.1445-2197.1981.tb04972.x

[47] S. Pohjola-Sintonen, P. Ruutu and K. Tallroth, "Hematogenous Candida Spondylitis. A Case Report,” Acta Medica Scandinavica, Vol. 215, No. 1, 1984, pp. 85-87. doi:10.1111/j.0954-6820.1984.tb04974.x

[48] M. J. Burton, P. Shah and E. Swiatlo, "Misidentification of Candida Parapsilosis as C Famata in a Clinical Case of Vertebral Osteomyelitis," American Journal of the Medical Sciences, Vol. 341, No. 1, 2011, pp. 71-73. doi:10.1097/MAJ.0b013e3181f54dab

[49] J. D. Morrow and F. A. Manian, "Vertebral Osteomyelitis Due to Candida Glabrata. A Case Report," Journal of Tennessee Medical Association, Vol. 79, No. 7, 1986, pp. 409-410.

[50] C. J. O’Connell, A. V. Cherry and J. G. Zoll, "Letter: Osteomyelitis of Cervical Spine: Candida Guilliermondii," Annals of Internal Medicine, Vol. 79, No. 5, 1973, p. 748. doi:10.7326/0003-4819-79-5-748_1

[51] F. J. Eismont, H. H. Bohlman, P. L. Soni, V. M. Goldberg and A. A. Freehafer, "Pyogenic and Fungal Vertebral Osteomyelitis with Paralysis," The Journal of Bone \& Joint Surgery. American Volume, Vol. 65, No. 1, 1983, pp. 1929.
[52] P. Rossel, H. C. Schonheyder and H. Nielsen, "Fluconazole therapy in Candida Albicans Spondylodiscitis," Scandinavian Journal of Infectious Diseases, Vol. 30, No. 5, 1998, pp. 527-530. doi:10.1080/00365549850161601

[53] I. G. de Matos, G. do Carmo and M. L. Araujo, "Spondylodiscitis by Candida Albicans,” Infection, Vol. 26, No. 3, 1998, pp. 195-196. doi:10.1007/BF02771856

[54] V. Boix, J. Tovar and A. Martin-Hidalgo, "Candida Spondylodiscitis. Chronic Illness Due to Heroin Analgesia in an HIV Positive Person,” The Journal of Rheumatology, Vol. 17, No. 4, 1990, pp. 563-565.

[55] W. Herzog, J. Perfect and L. Roberts, "Intervertebral Diskitis Due to Candida Tropicalis," Southern Medical Journal, Vol. 82, No. 2, 1989, pp. 270-273. doi:10.1097/00007611-198902000-00029

[56] K. J. Liudahl and T. J. Limbird, “Torulopsis Glabrata Vertebral Osteomyelitis. Case Report and Review of the Literature," Spine, Vol. 12, No. 6, 1987, pp. 593-595.

[57] I. F. Rowe, E. D. Wright, C. S. Higgens and J. P. Burnie, "Intervertebral Infection Due to Candida Albicans in an Intravenous Heroin Abuser," Annals of Rheumatic Diseases, Vol. 47, No. 6, 1988, pp. 522-525. doi:10.1136/ard.47.6.522

[58] D. L. Turner, S. A. Johnson and S. A. Rule, "Successful Treatment of Candidal Osteomyelitis with Fluconazole Following Failure with Liposomal Amphotericin B," Journal of Infection, Vol. 38, No. 1, 1999, pp. 51-53. doi:10.1016/S0163-4453(99)90032-4

[59] R. S. Holzman and F. Bishko, "Osteomyelitis in Heroin Addicts,” Annals of Internal Medicine, Vol. 75, No. 5, 1971, pp. 693-696. doi:10.7326/0003-4819-75-5-693

[60] S. Jonnalagadda, M. P. Veerabagu, J. Rakela, S. Kusne, P. Randhawa and M. Rabinovitz, "Candida Albicans Osteomyelitis in a Liver Transplant Recipient: A Case Report and Review of the Literature," Transplantation, Vol. 62, No. 8, 1996, pp. 1182-1184. doi:10.1097/00007890-199610270-00028

[61] K. Cho, S. H. Lee, E. S. Kim and W. Eoh, "Candida Parapsilosis Spondylodiscitis after Lumbar Discectomy,” Journal of Korean Neurosurgical Society, Vol. 47, No. 4, 2010, pp. 295-297. doi:10.3340/jkns.2010.47.4.295

[62] H. H. Moon, J. H. Kim, B. G. Moon and J. S. Kim, "Cervical Spondylodiscitis Caused by Candida Albicans in Non-Immunocompromised Patient,” Journal of Korean Neurosurgical Society, Vol. 43, No. 1, 2008, pp. 45-47. doi:10.3340/jkns.2008.43.1.45

[63] L. Seravalli, D. Van Linthoudt, C. Bernet, A. de Torrente, O. Marchetti, F. Porchet, et al., "Candida Glabrata Spinal Osteomyelitis involving Two Contiguous Lumbar Vertebrae: A Case Report and Review of the Literature," Diagnostic Microbiology \& Infectious Disease, Vol. 45, No. 2, 2003, pp. 137-141. doi:10.1016/S0732-8893(02)00497-2 